\title{
Piperacillin Anhydrous
}

National Cancer Institute

\section{Source}

National Cancer Institute. Piperacillin Anhydrous. NCI Thesaurus. Code C61891.

The anhydrous form of piperacillin, a broad-spectrum semisynthetic ureidopenicillin antibiotic. Piperacillin binds to penicillin binding proteins (PBP) located on the inner membrane of the bacterial cell wall, thereby interfering with the cross-linking of peptidoglycan chains necessary for bacterial cell wall streng th and rigidity. As a result, cell wall synthesis is interrupted leading to a weakened cell wall and eventually cell lysis. 